\section{Effect of cigarette smoking on subsequent postoperative thromboembolic disease in gynaecological patients}

The risk of deep vein thrombosis (DVT) in women taking oral contraceptives is increased if they are also cigarette smokers. ${ }^{1}$ On the other hand, cigarette smoking has been said to "protect" against the subsequent development of DVT after myocardial infarction ${ }^{23}$ and also after emergency surgery. ${ }^{4}$ Our own study into the relation between cigarette smoking and postoperative DVT was part of a multi-variable study of both clinical and coagulation factors in the prediction of a tendency to postoperative DVT. ${ }^{5}$

\section{Patients, methods, and results}

A total of 231 patients undergoing major gynaecological surgery were investigated. Their smoking habits over the month before surgery were established, and their weights and heights were also recorded. All patients underwent preoperative isotopic scanning of the legs using the ${ }^{125} \mathrm{I}$-fibrinogen technique. Routine postoperative scanning was performed on the first, third, and sxith day, unless a significantly high count was obtained, when daily scanning was started. None of the patients in this series received specific prophylaxis for DVT.

There were no patients with preoperative evidence of DVT. Postoperatively $37\left(16^{\circ}\right)$ of the 231 patients developed isotopic evidence of DVT. Of the 99 smokers, eight $(8.1 \%)$ developed DVT, while $29(22 \%)$ of the 132 non-smokers developed DVT (see table). The difference between the two proportions is significant at the $5 \%$ level on a $\chi^{2}$ test. The percentage overweight for height was calculated (Geigy tables) and showed that the mean percentage overweight for height in those with DVT was 13.0 compared with 4.8 in those without $(P<0 \cdot 05)$. When, however, the smokers and the non-smokers were considered in relation to their percentage overweight for height, we found that the eight patients who smoked and developed postoperative DVT had a mean percentage overweight for height of $18.7 \%$. The 91 smokers without DVT were only 3.7\% overweight for height. Of the non-smoking patients, 29 were in the DVT-positive group and were $11.4 \%$ overweight for height, compared with 103 in the DVT-negative group, who were only $5.8 \%$ overweight.

\section{Comment}

We found that cigarette smokers had a decreased incidence of postoperative DVT. Cigarette smoking increases the chances of developing myocardial infarction, but cigarette smokers who do suffer myocardial infarction seem to be less likely to develop complicating peripheral vein thrombosis. ${ }^{3}$ Our results also show an apparent protective value of cigarette smoking, since only $21^{\circ}$ of the DVTpositive group were cigarette smokers compared with $47^{\circ} \%$ of the DVT-negative group. The series differs from that of Pollock, ${ }^{4}$ in that all our patients had surgery performed as an elective procedure.

Deep vein thrombosis must be considered a disease of multifactorial origin. We have reported significant differences in certain clinical features and the results of preoperative coagulation tests between patients who postoperatively did or did not develop DVT. ${ }^{5}$ Our results show that the DVT-positive patients were significantly heavier than the DVT-negative ones. Smoking seemed to "protect" the patient against developing postoperative DVT. The results also show that the cigarette smokers who developed postoperative DVT were grossly overweight. The non-smoking DVT-positive patients were also overweight, but not to the same extent as the smoking group. It is tempting to suggest, therefore, that the overweight cigarette smoker runs a higher risk of developing postoperative DVT compared with the smoker who is close to optimum weight.

The explanation for this "protection" of our smoking patients against postoperative DVT provided they are lean remains obscure. No data exist to suggest that this is a direct function of cigarette smoking; it may be that the low incidence of postoperative DVT in non-obese cigarette smokers reflects resistance to venous thrombosis as a constitutional feature, which correlates with but is not due to a predilection to smoke cigarettes.

' Royal College of General Practitioners, Lancet, 1977, 2, 727.

${ }^{2}$ Handley, A J, and Teather, D, British Medical fournal, 1974, 3, 230.

${ }^{3}$ Marks, P, and Emerson, P A, British Medical fournal, 1974, 3, 232.

${ }^{4}$ Pollock, A V, British Medical fournal, 1974, 3, 522.

${ }^{5}$ Clayton, J K, et al, British Medical fournal, 1976, 2, 910.

(Accepted 10 April 1978)

Departments of Obstetrics and Gynaecology, Community Medicine $\frac{\rho}{\vec{D}}$ and General Practice, and Medicine, University of Leeds, Leeds $\mathbb{\mathbb { \Phi }}$ LS1 3EX

J K CLAYTON, MRCOG, senior registrar in obstetrics and gynaecology (now consultant obstetrician and gynaecologist, St Luke's Hospital, $\vec{O}$ Bradford)

J A ANDERSON, MA, DPHIL, (now reader in statistics, University of $\overrightarrow{\vec{\omega}}$ Newcastle upon Tyne)

G P MCNICOL, MD, PHD, professor of medicine

\section{Joint hypermobility mimicking pauci-articular juvenile polyarthritis}

Patients with generalised joint hypermobility may develop premature $\frac{c}{\mathrm{C}}$ osteoarthrosis and chondrocalcinosis. ${ }^{1}$ Nevertheless, symptoms from hypermobile joints occur as early as childhood, ${ }^{2}$ when the synovitis $\vec{\theta}$ associated with hypermobility may be confused with juvenile poly- $\infty$ arthritis. ${ }^{3}$ We describe a patient in whom symptoms initially attributed to chronic polyarthritis are more likely to be related to hypermobile joints.

\section{Case report}

A 13-year-old girl experienced pain and swelling, acute in onset, in the $\overrightarrow{\overline{0}}$ right knee after a race at school. She was talented at sports and dancing. A 3 few weeks later the proximal interphalangeal joint of her right middle finger became swollen and a month later she had swelling at the interphalangeal joint of the right thumb. She was referred to hospital within three months, when the presence of swelling was confirmed in these joints though of these only the right knee was hot, with a small effusion and some synovial thickening. The effusion was not aspirated. Haemoglobin concentration was $12 \cdot 4 \mathrm{~g} / \mathrm{dl}$, WBC $6000 \cdot 10^{9} / \mathrm{l}$, and ESR $9 \mathrm{~mm} /$ hour. $X$-ray films of the hands, cervical spine, and knees were normal and the latex fixation test result was negative. There was no history of sore throat and ASO titre was $1 / 50$. There was no evidence of carditis or systemic upset.

She was treated with soluble aspirin and rest and over three months all symptoms and signs disappeared. They have not recurred over the two years of follow-up in spite of return to normal activities. Her ESR has been N consistently normal, the ANF test result is negative, and findings on ophthalmological examination have been normal. The results of tissue typing were HLA A2,3; B7, 15.

The patient had always considered herself "double-jointed." She had a Carter and Wilkinson score (modified by Beighton ${ }^{4}$ ) of $9 / 9$ denoting 0

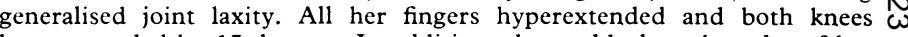
hyperextended by 15 degrees. In addition, she could place the soles of her $O$ feet together, extend both elbows backwards in the horizontal plane to $110^{\circ}$, and had lax feet and hands. Three siblings, none of them loose-jointed, had been free of rheumatic complaints. Nevertheless, the mother who had $\mathbb{D}$ experienced very similar symptoms to her daughter at the age of 14 , had been loose-jointed and still retained this feature in her hands and feet. She $T$ had also had severe varicose veins and crepitus in both knee joints at the age of 40. A maternal cousin and her daughter also considered themselves "double-jointed."

\begin{tabular}{|c|c|c|c|c|c|c|c|c|c|c|c|c|}
\hline & \multicolumn{6}{|c|}{ DVT-positive } & \multicolumn{6}{|c|}{ DVT-negative } \\
\hline & \multicolumn{3}{|c|}{ Smoker } & \multicolumn{3}{|c|}{ Non-smoker } & \multicolumn{3}{|c|}{ Smoker } & \multicolumn{3}{|c|}{ Non-smoker } \\
\hline & Mean & SE & No & Mean & SE & No & Mean & SE & No & Mean & SE & No \\
\hline$\%$ Overweight & $18 \cdot 7$ & 8.5 & 8 & $11 \cdot 4$ & $3 \cdot 2$ & 29 & $3 \cdot 7$ & 1.5 & 91 & $5 \cdot 8$ & $1 \cdot 3$ & 103 \\
\hline
\end{tabular}

